\title{
Arthroscopic-Assisted Removal of Proximal Humerus Locking Plates With Capsular Release Significantly Improves Range of Motion and Function
}

\author{
Justin M. Chan, B.A., Cynthia Kahlenberg, M.D., Naomi E. Gadinsky, M.D., \\ Dean G. Lorich, M.D., and Joshua S. Dines, M.D.
}

\begin{abstract}
Purpose: To investigate the clinical outcomes following the arthroscopic removal of proximal humerus locking plates for symptomatic hardware after open reduction and internal fixation (ORIF) of proximal humerus fractures. Methods: Patients who underwent arthroscopic removal of hardware (ROH) with capsular release due to pain and/or immobility after receiving locking plates to treat proximal humerus fractures from 2009 to 2016 were identified. Operative and clinic records were reviewed to obtain demographic information, concomitant procedures during $\mathrm{ROH}$, and pre- and postoperative active shoulder range of motion. Postoperative patient-reported outcomes included the QuickDASH, PROMIS Pain Intensity, Constant, and University of California, Los Angeles shoulder rating scale. Results: In total, 88 patients were included. Patients were evaluated at a minimum of 6 weeks postoperatively after ROH. Patients with pre- and postoperative active range of motion values demonstrated significant improvements in mean forward elevation $\left(\mathrm{n}=69 ; 78.4 \% ; 115.1^{\circ}\right.$ to $\left.152.1^{\circ}, P<.001\right)$, abduction $(\mathrm{n}=29$; $33.0 \% ; 70.9^{\circ}$ to $\left.138.7^{\circ}, P<.001\right)$, external rotation $\left(\mathrm{n}=49 ; 55.7 \% ; 43.7^{\circ}\right.$ to $\left.58.6^{\circ}, P=.012\right)$, and internal rotation $\left(\mathrm{n}=45 ; 51.1 \% ; 25.7^{\circ}\right.$ to $\left.61.9^{\circ}, P<.001\right)$. Patients also reported positive patient-reported scores, including the QuickDASH $(4.1 \pm 7.8)$, PROMIS Pain Intensity $(3.5 \pm 0.9)$, Constant $(84.6 \pm 10.7)$, and University of California, Los Angeles shoulder rating scale $(33 \pm 2.9)$, which were measured $70.6 \pm 26.6$ months postoperatively. There were no surgical complications, no arthroscopic cases were converted to open, but 2 reported refractures $(2.3 \%)$. Conclusions: Arthroscopic-assisted removal of proximal humerus locking plates significantly improves motion and function while allowing for management of concomitant shoulder pathology and potentially avoiding open surgery complications. Given that patients undergoing this procedure frequently have multiple comorbidities, arthroscopic-assisted removal with smaller incisions may minimize risks while restoring shoulder mobility. Therefore, arthroscopic ROH for patients experiencing symptomatic hardware after ORIF is recommended. Level of Evidence: Level IV, therapeutic case series.
\end{abstract}

$\mathbf{L}$ ocking plates for open reduction and internal fixation (ORIF) of proximal humerus fractures provide positive outcomes in most cases. ${ }^{1-3}$ However, numerous studies have reported the potential for

From the Department of Sports Medicine and Shoulder Surgery, Hospital for Special Surgery, New York, New York, USA.

The authors report the following potential conflicts of interest or sources of funding: This project was supported in part by funds from the Clinical Translational Science Center (CTSC), National Center for Advancing Translational Sciences (NCATS) grant \#1-UL1-TR002384-01. The content is solely the responsibility of the authors and does not necessarily represent the official views of the funding source NCATS, based in Rockville, MD, J.S.D. reports personal fees and other from Arthrex, personal fees from Trice, personal fees from Intellectual Property from CONMED Linvatec, personal fees from the American Journal of Orthopedics, and personal fees from the Journal of Shoulder and Elbow Surgery, outside the submitted work. Full ICMJE author disclosure forms are available for this article online, as supplementary material. complications associated with proximal humerus locking plates, such as malreduction, malunion, nonunion, infection, as well as issues from primary and secondary screw perforations into the glenohumeral joint. ${ }^{1,4-11}$

The coinvestigators acknowledge the significant contributions to this project of Dean G. Lorich, M.D., prior to his passing on December 10, 2017.

Received January 3, 2020; accepted September 13, 2020.

Address correspondence to Joshua S. Dines, M.D., Hospital for Special Surgery, 610 W 58th St., 3rd Floor, New York, NY 10019. E-mail: jdinesmd@gmail.com

(C) 2020 THE AUTHORS. Published by Elsevier Inc. on behalf of the Arthroscopy Association of North America. This is an open access article under the CC BY-NC-ND license (http://creativecommons.org/licenses/by-nc-nd/4.0/). 2666-061X/191575

https://doi.org/10.1016/j.asmr.2020.09.013 
The initial fracture pattern is also a significant factor, with 3- and 4-part fractures associated with a high rate of complications compared with 2- and 3- part fractures. ${ }^{11-13}$ In addition, many patients without complications fail to regain full range of motion (ROM) and/or have persistent postoperative pain. For these patients, removal of hardware $(\mathrm{ROH})$ and capsular release may improve shoulder function. ${ }^{14-18}$ Other revision procedures besides hardware removal include hemiarthroplasty, reverse shoulder arthroplasty, and total shoulder arthroplasty.

Hardware removal can be done via an open or arthroscopic approach. Open ROH requires a larger incision and makes visualization of intra-articular pathology more difficult compared with arthroscopic surgery. Complications associated with open $\mathrm{ROH}$ include the risk of refracture, infection, axillary nerve damage, increased risk of bleeding, and wound healing complications from the large incision. ${ }^{19-23}$

To help overcome the complications associated with retained hardware and open $\mathrm{ROH}$, arthroscopic-assisted $\mathrm{ROH}$ has been described. Dines et al. ${ }^{24}$ previously detailed the technique for arthroscopic-assisted hardware removal following ORIF of proximal humerus fractures, which describes posterior, anteroinferior, and anterolateral standard portals used. At the time of incision, the lateral portal is extended by about $1.5 \mathrm{~cm}$, so a there is a $2.5-$ to $3-\mathrm{cm}$ incision proximally and then under direct arthroscopic visualization, a distal incision that measures $2.5 \mathrm{~cm}$ is centered over the 3 distal screws used in the plate. Since that time, several series have reported improvements in ROM and positive patient-reported outcome scores after open $\mathrm{ROH}^{14,15}$ and arthroscopic ROH. ${ }^{16-18}$ Considering patients with extensive concomitant articular pathologies often present in proximal humerus fracture patient populations, ${ }^{18,25}$ arthroscopic $\mathrm{ROH}$ may be preferable to open $\mathrm{ROH}$ due to its minimally invasive nature as well as its ability to address concomitant pathology.

The purpose of this study was to investigate the clinical outcomes following the arthroscopic removal of proximal humerus locking plates for symptomatic hardware after ORIF of proximal humerus fractures. The hypothesis of this study is that arthroscopic removal of hardware with capsular release would lead to significant improvements in ROM and patientreported outcome scores with a low complication rate.

\section{Methods}

This was a retrospective case series of patients who had previously undergone ORIF for proximal humerus fractures who elected to undergo removal of proximal humerus locking plates with capsular release from January 2009 to December 2016 at a single institution. The proximal humerus fractures eligible for the study were 2-, 3-, and 4-part fractures. Exclusion criteria included patients who were converted to prosthetic shoulder replacement in conjunction with hardware removal, patients who underwent $\mathrm{ROH}$ for fracture collapse, and patients who lacked a 6-week minimum follow-up from the removal procedure. The minimum was 6 weeks due to patients commonly cancelling follow-up appointments if they were asymptomatic after approximately 2 months postoperatively. This study was approved by the Hospital for Special Surgery Clinical Review Panel and Institutional Review Board (study ID: 2018-0533).

All ORIFs were performed by a single orthopaedic trauma fellowship-trained surgeon, and all arthroscopic ROH procedures were performed by another fellowship-trained sports medicine and shoulder specialist surgeon. Indications for arthroscopic instead of open $\mathrm{ROH}$ were surgeon preference due to added benefits of using a diagnostic scope and addressing concomitant pathology. Patients qualified for elective plate removal if they were deemed to be radiographically healed via radiographs or computed tomography before plate removal, and had plateaued with regards to progress with physical therapy, yet still experienced significant lack of ROM, persistent pain in the region of the plate that limited daily activities, and/or infection.

\section{Preoperative Assessment}

Operative and clinic records were reviewed to obtain the reason for hardware removal, Neer classification of the original fracture, fracture laterality, hand dominance, and concomitant procedures at the time of $\mathrm{ROH}$. Preoperative (pre-ROH) active shoulder ROM was collected via goniometer by the operating surgeon, including forward flexion, abduction, external rotation, and internal rotation.

\section{Postoperative Assessment}

At the most recent follow-up, postoperative (post$\mathrm{ROH}$ ) active shoulder ROM was collected via goniometer by the operating surgeon. Postoperative patient-reported outcome scores, which included the QuickDASH, PROMIS Pain Intensity, Constant, and University of California, Los Angeles (UCLA) shoulder rating scale, were obtained either during the most recent follow-up or by telephone interview. The ROM values for the Constant and UCLA scores were extrapolated from the postoperative clinic notes at the most recent follow-up. Results from these questionnaires and surveys were recorded in REDCap, the study's institution's secure database for compiling patient-reported outcomes. Postoperative complications including infection, refracture, and subsequent revision shoulder surgery, such as conversion to reverse shoulder arthroplasty were recorded. 


\section{Surgical Technique}

Initial ORIF was performed with precontoured Synthes Proximal Humerus Locking Plates (Synthes, Paoli, PA) (Fig 1). The arthroscopic ROH technique has been previously described. ${ }^{24}$ To summarize, posterior, anteroinferior, and anterolateral standard portals were used. At the time of removal, concomitant shoulder pathology was addressed arthroscopically, including capsular release and subacromial decompression. Specifically, for a right shoulder, capsular release was done in standard fashion with ArthroCare (Stryker, Kalamazoo, MI) starting with the anterior capsule extending inferiorly to about the 5:30 PM relative to the face of the glenoid after which the arthroscope was switched to the rotator interval portal and the posterior capsule was released starting just posterior to the biceps anchor extending inferior to about the 7-o'clock position relative to the face of the glenoid. After initial diagnostic arthroscopy, dissection around the plate was performed arthroscopically and the axillary nerve was visualized and neurolyzed in all cases. The screw under the nerve was removed through an accessory lateral portal. Distal screws were removed through one distal localized portal. The plate and proximal screws were removed through the lateral portal. The plate was then gently elevated from the bone and brought up through the lateral portal (Fig 2). ${ }^{24,26}$

\section{Postoperative Protocol}

Postoperative protocols involved patients wearing a sling for comfort for the first 4 to 6 weeks. Aggressive passive and active-assisted ROM in all planes were initiated on postoperative day 1. Patients progressed to strengthening exercises at week 4 .

\section{Statistical Analysis}

Pre- and postoperative ROM values were determined to have non-normal distributions using the Shapiro-Wilk test; therefore, nonparametric testing was performed using the Wilcoxon signed-rank test to compare pre- and postoperative ROM. Secondary outcomes included postoperative patient-reported outcomes scores (QuickDASH, PROMIS Pain Intensity, Constant, and UCLA), which were reported using means and standard deviations. Statistical significance was set at $P<.05$. Statistical analysis was performed using SPSS 22 (IBM, Armonk, NY).

\section{Results}

A total of 88 patients ( 22 men and 66 women at an average age of $59 \pm 13.5$ years at the time of surgery) met inclusion criteria and were included in the study, with $22(25 \%)$ lost to follow-up due to not answering the questionnaire, refusing to participate, or death
(Table 1). Mean body mass index was $28.2 \pm 6.5,9$ patients $(10.2 \%)$ had diabetes, and $4(4.5 \%)$ were active smokers. There were $45(51.1 \%)$ fractures on the right arm. $66(75 \%)$ patients had recorded Neer classifications for their initial proximal humerus fractures; Neer type 2 in $15(22.7 \%)$, type 3 in $21(31.8 \%)$, and type 4 in $30(45.5 \%)$ patients. Four patients $(4.5 \%)$ had avascular necrosis (AVN) of the humeral head before $\mathrm{ROH}$. The mean duration from proximal humerus ORIF to $\mathrm{ROH}$ was $11.4 \pm 10.2$ months (range, 3.9-64.2 months), with only 1 patient undergoing $\mathrm{ROH}<6$ months after ORIF. A total of 18 patients $(20.5 \%)$ underwent ROH due to pain only, $19(21.6 \%)$ due to lack of ROM only, $50(56.8 \%)$ due to both pain and lack of ROM, and $1(1.1 \%)$ due to infection. All patients had prominent hardware superior laterally on diagnostic arthroscopy and underwent axillary nerve neurolysis at the time of $\mathrm{ROH}$, as well as capsular release and subacromial decompression. Five patients $(5.7 \%)$ had secondary screw perforations based on direct view intra-articularly with the scope. Other concomitant procedures included biceps scope tenotomy/tenodesis (14 patients; $15.9 \%$ ), removal of loose bodies from joint (7 patients; $8.0 \%$ ), humeral head/glenoid chondroplasty (12 patients; $13.6 \%$ ), rotator cuff repair or debridement (9 patients; $10.2 \%$ ), and labral repair or debridement ( 5 patients; $5.7 \%$ ). All patients were evaluated at a minimum of 6 weeks postoperatively. The mean time from $\mathrm{ROH}$ to the latest follow-up was $4.7 \pm 7$ months (range, 1.5-52.4 months).

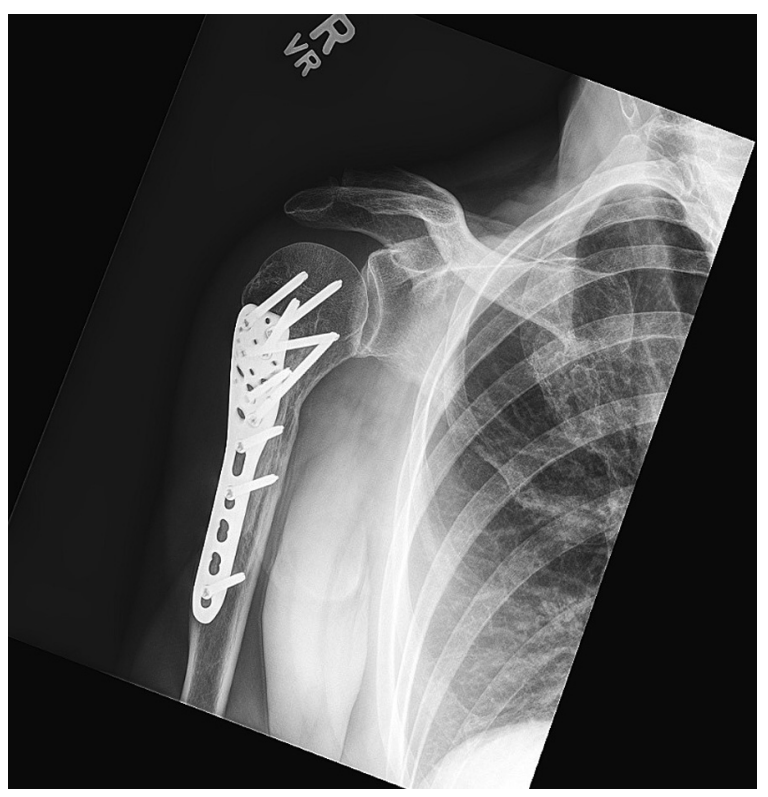

Fig 1. Radiograph of the right proximal humerus after initial open reduction internal fixation with a precontoured locking plate. 


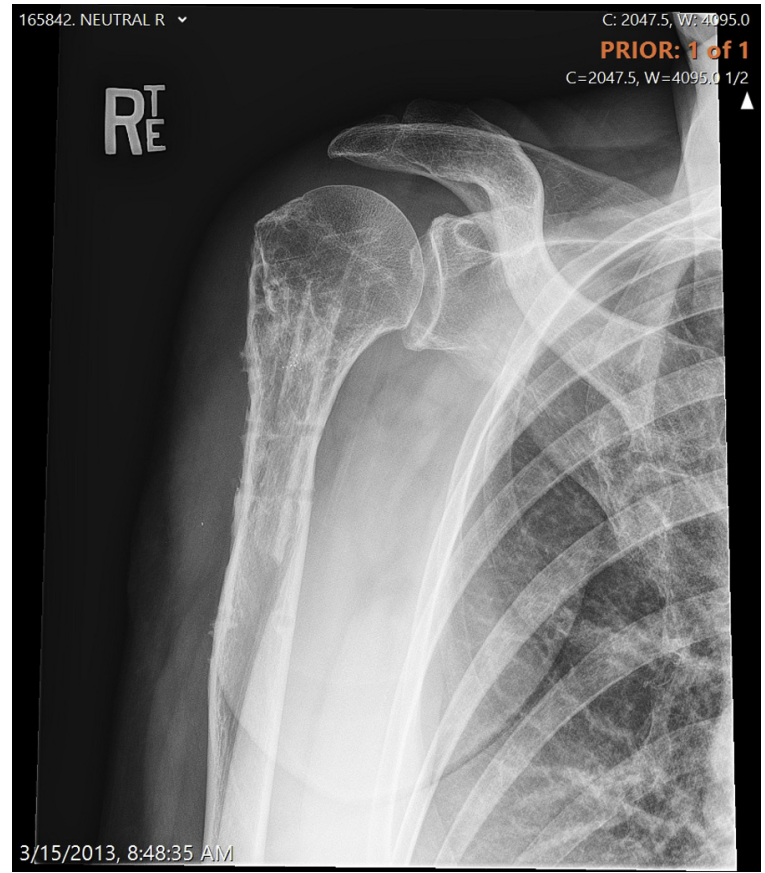

Fig 2. Radiograph of the right proximal humerus status postremoval of precontoured locking plate 64 months after initial open reduction internal fixation surgery with a fibular strut.

\section{Clinical and Functional Evaluation Results}

Pre- and postoperative active shoulder ROM values were available for a subset of patients for forward flexion ( $\mathrm{n}=69 ; 78.4 \%)$, abduction $(\mathrm{n}=29 ; 33.0 \%)$, external rotation $(\mathrm{n}=49 ; 55.7 \%)$, and internal rotation $(\mathrm{n}=45 ; 51.1 \%)$. There were significant improvements in mean forward elevation $\left(115.1^{\circ}\right.$ to $\left.152.1^{\circ}, P<.001\right)$, abduction $\left(70.9^{\circ}\right.$ to $\left.138.7^{\circ}, P<.001\right)$, external rotation $\left(43.7^{\circ}\right.$ to $\left.58.6^{\circ}, P=.012\right)$, and internal rotation $\left(25.7^{\circ}\right.$ to $61.9^{\circ}, P<.001$ ) (Table 2). In total, $87 \%$ of patients had minimal pain or no pain at 6-weeks' follow up.

The number of patients with available postoperative outcome scores was 66 (75\%). Postoperative patientreported scores included the QuickDASH (mean $4.1 \pm$ 7.8; range, 2.3-45.5), PROMIS Pain Intensity (3.5 \pm 0.9 ; range, 3-7), Constant ( $84.6 \pm 10.7$; range, $43-100)$, and UCLA (33 \pm 2.9 ; range, 22-35). The scores were measured at a mean of $70.6 \pm 26.6$ months (range, 36.6-125.6 months) after ROH (Table 3).

The revision rate was $2.3 \%$, with 2 patients suffering refractures, one experiencing the refracture of the humeral shaft through one of the inferior screw holes and the other experiencing a traumatic refracture after a fall. Each required revision procedures, specifically a revision ORIF and a hemiarthroplasty, respectively. There were no other complications, including infection, wound dehiscence, or nerve issues in this case series, and no arthroscopic cases were converted to open.

\section{Discussion}

The findings of this study confirm our hypothesis that elective arthroscopic plate removal after ORIF of proximal humerus fractures leads to improvements in ROM and functional outcomes with minimal complications. The arthroscopic ROH produced excellent results, including positive clinical outcomes shown by the patient-reported outcome scores and a low overall complication rate of $2.3 \%$.

The study's clinical outcomes are largely in line with previous ROH outcome reports. Acklin et al. ${ }^{14}$ reported a series of 20 patients whose Constant scores significantly improved from a mean of 71 to 76 following open $\mathrm{ROH}$ with no intraoperative complications. Although preoperative Constant scores were not obtained, the postoperative Constant scores of the cohort in this study were greater at $84.6 \pm 10.7$. Richards et al. ${ }^{27}$ and Kirchhoff et al. ${ }^{15}$ observed considerable clinical improvements in patients following open $\mathrm{ROH}$ but could not justify a general recommendation for $\mathrm{ROH}$ in asymptomatic patients due to the surgical and anesthetic risks associated with open surgery. Furthermore, any scar tissue in the rotator interval can be addressed during arthroscopic $\mathrm{ROH}$ through a deltopectoral interval while using a deltoid split for fixation (Fig 3).

Maqdes et al. ${ }^{16}$ reviewed 11 patients who underwent the arthroscopic $\mathrm{ROH}$ and reported significant improvements in pain, ROM, and all functional parameters with no infections or wound dehiscence.

Table 1. Baseline Patient Characteristics

\begin{tabular}{lc}
\hline & Arthroscopic \\
\hline $\mathrm{n}$ & 88 \\
Age, years & $59 \pm 13.5$ \\
Male sex & $22(25 \%)$ \\
Right shoulder & $45(51.1 \%)$ \\
BMI & $28.2 \pm 6.5$ \\
Neer classification & $\mathrm{N}=66(75 \%)$ \\
2 part & $15(22.7 \%)$ \\
3 part & $21(31.8 \%)$ \\
4 part & $30(45.5 \%)$ \\
Active smoker & $4(4.5 \%)$ \\
Diabetes & $9(10.2 \%)$ \\
AVN & $4(4.5 \%)$ \\
ROH reason* & $18(20.5 \%) ; 19(21.6 \%) ; 50(56.8 \%) ;$ \\
& $1(1.1 \%)$ \\
ORIF to ROH $\dagger \dagger$ & $11.4 \pm 10.2 ; 3.9-64.2$ \\
ROH to Last FU $\dagger \ddagger$ & $4.7 \pm 7 ; 1.5-52.4$ \\
Lost to FU & $22(25 \%)$ \\
\hline
\end{tabular}

NOTE. "Right shoulder" indicates the number of plate removals done on right shoulders.

AVN, avascular necrosis of the humeral head; BMI, body mass index; FU, follow-up; ORIF, open reduction and internal fixation; $\mathrm{ROH}$, removal of hardware; ROM, range of motion.

*Pain; lack of ROM; both pain and lack of ROM; infection.

${ }^{\dagger}$ Months.

${ }^{\ddagger}$ Average \pm standard deviation; minimum-maximum. 
Table 2. Range of Motion Results

\begin{tabular}{lcrrr}
\hline & $\mathrm{n}$ & Preop Status & \multicolumn{2}{c}{ Postop Status } \\
\hline Active forward elevation, & 69 & $115.1 \pm 35.8$ & $152.1 \pm 25$ & $<.001$ \\
Active abduction, & 29 & $70.9 \pm 18.7$ & $58.7 \pm 25.4$ & $<.001$ \\
Active external rotation, & 49 & $43.7 \pm 25.4$ & $61.9 \pm 10.6$ & .012 \\
Active internal rotation, & 45 & $25.7 \pm 23.8$ & $<.001$ \\
\hline
\end{tabular}

NOTE. Values are average \pm standard deviation.

Preop, latest preoperative range of motion measurement before removal of hardware; Postop, final follow-up range of motion measurement after surgery.

With one of the largest cohorts to date, Maroun et al. ${ }^{17}$ reported an improvement in all active ROM parameters, overall function, and pain levels for 58 patients at the latest follow-up after arthroscopic ROH. Katthagen et al. ${ }^{18}$ also observed significant improvements in active ROM for 45 patients after arthroscopic $\mathrm{ROH}$ and concluded that arthroscopy provides promising results in cases of high plate positioning, screw perforation, and postoperative or post-traumatic shoulder stiffness. None of these studies reported any complications.

There are many potential advantages of arthroscopic $\mathrm{ROH}$, including less peri- and postoperative morbidity, minimal soft-tissue trauma and/or blood loss, reduced risk of postoperative infections or adhesions, and complete glenohumeral inspection and treatment of concomitant intra-articular injuries. ${ }^{15,17,18,26}$ However, there are still associated risks. Richards et al. ${ }^{27}$ reported a 3\% complication rate including one refracture, one radial nerve injury, and one hematoma in patients undergoing removal of a variety of different metal internal fixation devices. Refracture rates after $\mathrm{ROH}$ have not been reported in current literature, but there were 2 refractures $(2.3 \%)$ in this study. The first patient, who had the hardware removed due to continued pain, developed AVN before $\mathrm{ROH}$, and refractured the humeral shaft through one of the inferior screw holes due to a fall 1 month following ROH. This patient ultimately underwent revision ORIF with another locking plate. The second patient, who had the hardware removed due to continued pain and lack of ROM, also developed AVN before $\mathrm{ROH}$, then suffered a traumatic refracture 6

Table 3. Postoperative Functional Outcome Scores

\begin{tabular}{lc}
\hline & Arthroscopic ROH \\
\hline $\mathrm{n}$ & 66 \\
QuickDASH & $4.1 \pm 7.8 ; 2.3-45.5$ \\
PROMIS Pain Intensity* $^{*}$ & $3.5 \pm 0.9 ; 3-7$ \\
Constant* $^{*}$ & $84.6 \pm 10.7 ; 43-100$ \\
UCLA $^{*}$ & $33 \pm 2.9 ; 22-35$ \\
Time $^{+*}$ & $70.6 \pm 26.6 ; 36.6-125.6$ \\
\hline ROH, removal of hardware; UCLA, University of California, LoS \\
Angeles shoulder rating scale. \\
$\quad \dagger$ Months from plate removal to answering the questionnaire. \\
$\quad$ *Average \pm standard deviation; minimum-maximum.
\end{tabular}

months following $\mathrm{ROH}$, and then progressed to a hemiarthroplasty 8 months post-ROH. These 2 complications are likely associated with the development of AVN after the initial trauma and ORIF, rather than with the arthroscopic technique itself. Schnetzke et al. ${ }^{28}$ observed a positive correlation between AVN and subsequent revision surgery after ORIF, noting a strong association between the degree of AVN progression and the amount of time elapsed from the initial injury to ORIF. Katthagen et al. ${ }^{18}$ also reported that patients with AVN of the humeral head exhibited poorer clinical outcomes following ORIF, requiring secondary arthroplasty implantation more frequently. Considering both refracture patients in the cohort developed AVN before $\mathrm{ROH}$, their complications may stem from the progression of AVN and may not be related to the $\mathrm{ROH}$ procedure. Although further research is needed to determine the optimal time for $\mathrm{ROH}$, the benefits of improved pain and ROM outweigh the risks in most cases.

\section{Limitations}

There are many limitations to this study. First, not all patients had pre- and postoperative ROM scores or responded to the questionnaire. However, 66 patients $(75 \%)$ still responded to the questionnaire. Next, the mean time from hardware removal to final office follow-up was short, approximately 5 months. This is due to patients not attending their follow-up appointments after their incisions were healed and their motion returned. Nonetheless, the time from $\mathrm{ROH}$ to completing the patient-reported outcome scores was $70.6 \pm 26.6$ months, and a previous study showed no clinical change during the second postoperative year. ${ }^{18}$ Subsequently, there was a discrepancy between the minimum time for the clinical ROM measurements and postoperative questionnaire of 6 weeks and 36 months, respectively, due to the retrospective nature of the study. In addition, only $75 \%$ of patients had a Neer fracture classification in the records. Also, the patients in this study underwent a number of concomitant procedures in addition to $\mathrm{ROH}$, which makes it difficult to quantify the benefit gained from hardware removal versus these other additional procedures. Furthermore, there were no preoperative patient-reported outcomes 


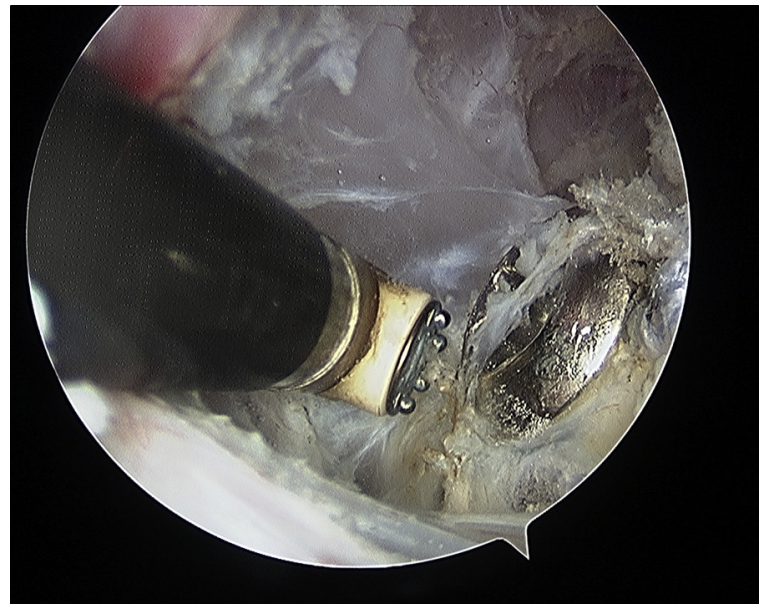

Fig 3. Arthroscopic photo from posterior portal with $70^{\circ}$ scope showing intra-articular debridement of scar tissue between the undersurface of the deltoid and plate during arthroscopic-assisted removal of proximal humerus plate.

scores, but it may be reasonably assumed the scores would be poor, considering all included patients elected for hardware removal due to pain and immobility. In addition, the complication rate may not be representative due to nonresponse bias of the patients lost to follow-up. Lastly, there was no open $\mathrm{ROH}$ comparison group, which would have elucidated the potential benefits of arthroscopic versus open ROH.

\section{Conclusions}

Arthroscopic-assisted removal of proximal humerus locking plates significantly improves motion and function while allowing for management of concomitant shoulder pathology and potentially avoiding open surgery complications. Given that patients undergoing this procedure frequently have multiple comorbidities, arthroscopic-assisted removal with smaller incisions may minimize risks while restoring shoulder mobility. Therefore, arthroscopic $\mathrm{ROH}$ for patients experiencing symptomatic hardware after ORIF is recommended.

\section{References}

1. Spross C, Platz A, Erschbamer M, Lattmann T, Dietrich M. Surgical treatment of neer group vi proximal humeral fractures: Retrospective comparison of PHILOS ${ }^{\circledR}$ and hemiarthroplasty. Clin Orthop Relat Res 2012;470: 2035-2042.

2. Khmelnitskaya E, Lamont LE, Taylor SA, Lorich DG, Dines DM, Dines JS. Evaluation and management of proximal humerus fractures. Adv Orthop 2012;2012:1-10.

3. Chudik SC, Weinhold P, Dahners LE. Fixed-angle plate fixation in simulated fractures of the proximal humerus: A biomechanical study of a new device. J Shoulder Elbow Surg 2003;12:578-588.

4. Berkes MB, Little MTM, Pardee NC, Schottel PC, Lazaro LE, Lorich DG. Outcomes of proximal humerus fracture open reduction internal fixation with concomitant ipsilateral shoulder girdle injuries: A case control study. HSS J 2016;12:105-110.

5. Brunner F, Sommer C, Bahrs C, et al. Open reduction and internal fixation of proximal humerus fractures using a proximal humeral locked plate: A prospective multicenter analysis. J Orthop Trauma 2009;23:163-172.

6. Jost B, Spross C, Grehn H, Gerber C. Locking plate fixation of fractures of the proximal humerus: Analysis of complications, revision strategies and outcome. J Shoulder Elbow Surg 2013;22:542-549.

7. Lovald S, Mercer D, Hanson J, et al. Complications and hardware removal after open reduction and internal fixation of humeral fractures. J Trauma 201 1;70:1273-1277.

8. Owsley KC, Gorczyca JT. Displacement/screw cutout after open reduction and locked plate fixation of humeral fractures. J Bone Joint Surg Am 2008;90:233-240.

9. Solberg BD, Moon CN, Franco DP, Paiement GD. Locked plating of 3- and 4-part proximal humerus fractures in older patients: The effect of initial fracture pattern on outcome. J Orthop Trauma 2009;23:113-119.

10. Südkamp N, Bayer J, Hepp P, et al. Open reduction and internal fixation of proximal humeral fractures with use of the locking proximal humerus plate. Results of a prospective, multicenter, observational study. J Bone Joint Surg Am 2009;91:1320-1328.

11. Spross C, Platz A, Rufibach K, Lattmann T, Forberger J, Dietrich M. The PHILOS plate for proximal humeral fractures-Risk factors for complications at one year. J Trauma Acute Care Surg 2012;72:783-792.

12. Neer CS. Displaced proximal humeral fractures. II. Treatment of three-part and four-part displacement. J Bone Joint Surg Am 1970;52:1090-1103.

13. Schulte LM, Matteini LE, Neviaser RJ. Proximal periarticular locking plates in proximal humeral fractures: Functional outcomes. J Shoulder Elbow Surg 2011;20: 1234-1240.

14. Acklin YP, Michelitsch C, Sommer C. Elective implant removal in symptomatic patients after internal fixation of proximal humerus fractures improves clinical outcome. BMC Musculoskelet Disord 2016;17:1-5.

15. Kirchhoff C, Braunstein V, Kirchhoff S, et al. Outcome analysis following removal of locking plate fixation of the proximal humerus. BMC Musculoskelet Disord 2008;9:138.

16. Maqdes A, Levy B, Klouche S, Hardy P. The feasibility and results of an arthroscopic removal of humeral locking plates and glenohumeral arthrolysis after proximal humeral fractures. Knee Surg Sport Traumatol Arthrosc 2014;22:456-461.

17. Maroun C, Aliani D, Hass A, Werthel JD, Vidil A, Valenti P. Shoulder arthroscopy combined to hardware removal in proximal humeral fractures: A series of 58 cases with a mean follow-up of 2 years. Eur J Orthop Surg Traumatol 2017;27:317-321.

18. Katthagen JC, Hennecke D, Jensen G, Ellwein A, Voigt C, Lill H. Arthroscopy after locked plating of proximal humeral fractures: Implant removal, capsular release, and intra-articular findings. Arthroscopy 2014;30: $1061-1067$.

19. Brown OL, Dirschl DR, Obremskey WT. Incidence of hardware-related pain and its effect on functional outcomes after open reduction and internal fixation of ankle fractures. J Orthop Trauma 2001;15:271-274. 
20. Böstman O, Pihlajamäki H. Routine implant removal after fracture surgery: A potentially reducible consumer of hospital resources in trauma units. J Trauma 1996;41:846-849.

21. Sanderson PL, Ryan W, Turner PG. Complications of metalwork removal. Injury 1992;23:29-30.

22. Raja S, Imbuldeniya AM, Garg S, Groom G. Difficulties encountered removing locked plates. Ann R Coll Surg Engl 2012;94:502-505.

23. Schwarz N, Euler S, Schlittler M, et al. Technical complications during removal of locking screws from locking compression plates: A prospective multicenter study. Eur J Trauma Emerg Surg 2013;39:339-344.

24. Dines JS, Hettrich CM, Kelly BT, Savoie FH, Lorich DG. Arthroscopic removal of proximal humerus locking plates. Knee Surg Sport Traumatol Arthrosc 2010;18: 409-411.

25. Schai PA, Hintermann B, Koris MJ. Preoperative arthroscopic assessment of fractures about the shoulder. Arthroscopy 1999; 15:827-835.

26. Voigt C, Geisler A, Lill H. Arthroscopic locking plate removal after proximal humeral fractures. Arch Orthop Trauma Surg 2010;130:391-395.

27. Richards RH, Palmer JD, Clarke NMP. Observations on removal of metal implants. Injury 1992;23:25-28.

28. Schnetzke M, Bockmeyer J, Loew M, Studier-Fischer S, Grützner PA, Guehring T. Rate of avascular necrosis after fracture dislocations of the proximal humerus: Timing of surgery. Obere Extrem 2018;13:273-278. 